\title{
Modeling the Laser Interferometer Space Antenna Optics
}

\author{
Eugene Waluschka ${ }^{a}$, Tracy R. Pedersen ${ }^{b}$, Paul McNamara $^{c}$ \\ ${ }^{a}$ NASA/Goddard Space Flight Center/551.0, Greenbelt, Maryland 20771 \\ ${ }^{b}$ Swales Aerospace, 5050 Powder Mill Rd, Beltsville, MD 20705 \\ 'University of Maryland, College Park, MD 20742
}

\begin{abstract}
The current approach to the creation of a computer model of the Laser Interferometer Space Antenna's (LISA) optical paths, which extend over 5 million kilometers, are discussed and future work outlined.

(C2005 Optical Society of America
\end{abstract}

OCIS codes: $(000.2780) ;(030.1670)$

\section{Summary}

The Laser Interferometer Space Antenna (LISA), shown below, will detect gravitational waves produced by objects such as binary black holes or objects falling into black holes (extreme mass ratio inspirals) over a frequency range of $10^{-4}$ to $0.1 \mathrm{~Hz}$. Within the conceptual frame work of Newtonian physics, a gravitational wave produces a strain, $\Delta l / l$, with magnitudes of the order of $10^{-21}$. Earth based gravitational wave detectors, such as the Laser Interferometer Gravitational-Wave Observatory (LIGO) project, use Michelson interferometers with arm lengths $l=4 \mathrm{~km}$ to detect these strains. Earth induced seismic noise limits ground-based instruments detecting gravitational waves with frequencies lower than $\sim 1 \mathrm{~Hz}$.

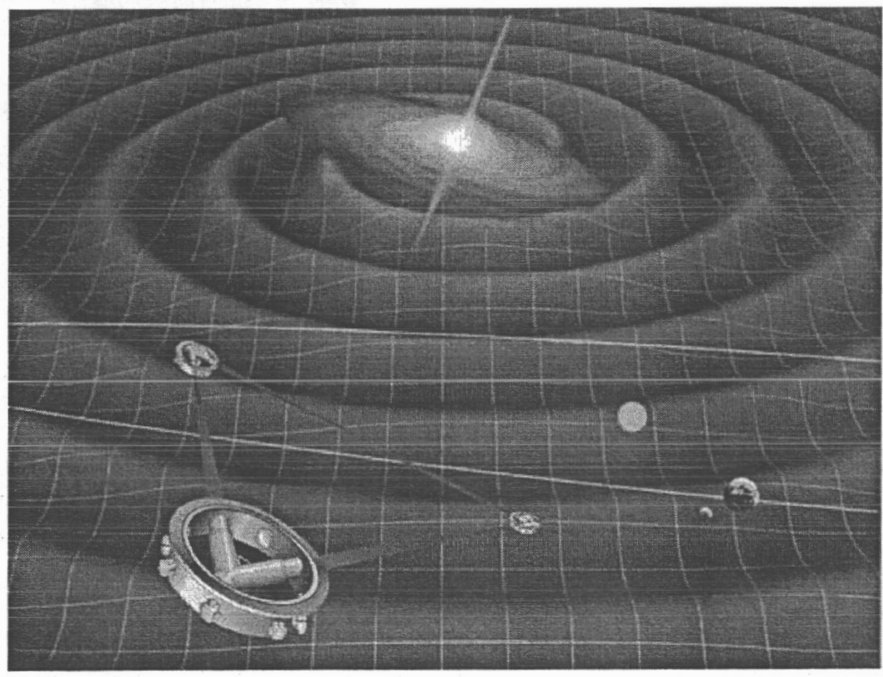

LISA, in a sense, consists of three Michelson-like interferometers modified to accommodate the dictates of orbital mechanics and the large distances. The armlengths are formed by three spacecraft in three separate orbits about the sun. The orbits are chosen such that the spacecraft maintain a large rotating equilateral triangular configuration of 5 million kilometers on a side. The interferometer, where the send and receive directions differ due to time of (light) flight effects, measures the changes in distance between freely falling proof masses in each spacecraft by suitable fringe counting techniques. The gravitational wave signal is extracted from the very slight variations from the expected fringe counting rates.

Creating an optical model of LISA which can be used to predict optical sensitivities and set tolerances, not only to traditional optical parameters, but also to control laws which orient the spacecraft and maintain nearly drag free motion of the proof masses, sufficiently well such that picometer level displacements (over 5 million kilometers) can be reliably seen is discussed. The current approach to creating this model(s) relies on existing optical design software and new software specifically tailored for LISA. Examples of current work are presented and discussed. 